\title{
Adherence to Pre-operative Exercise and the Response to Prehabilitation in Oesophageal Cancer Patients
}

\author{
Laura J. Halliday ${ }^{1} \cdot$ Emre Doganay $^{1} \cdot$ Venetia Wynter-Blyth ${ }^{2} \cdot$ Hayley Osborn $^{2} \cdot$ John Buckley $^{3} \cdot$ Krishna Moorthy $^{1}$
}

Received: 8 November 2019 / Accepted: 29 February 2020 / Published online: 20 April 2020

(C) 2020 The Author(s)

\begin{abstract}
Background Prehabilitation is thought to reduce post-operative respiratory complications by optimising fitness before surgery. This prospective, single-centre study aimed to establish the effect of pre-operative exercise on cardiorespiratory fitness in oesophageal cancer patients and characterise the effect of adherence and weekly physical activity on response to prehabilitation. Methods Patients received a personalised, home-based pre-operative exercise programme and self-reported their adherence each week. Cardiorespiratory fitness $\left(\mathrm{pVO}_{2} \mathrm{max}\right.$ and $\mathrm{O}_{2}$ pulse) was assessed at diagnosis, following completion of neoadjuvant chemotherapy (NAC) and immediately before surgery. Study outcomes included changes in fitness and post-operative pneumonia.

Results Sixty-seven patients with oesophageal cancer underwent prehabilitation followed by surgery between January 2016 and December 2018. Fitness was preserved during NAC and then increased prior to surgery ( $\mathrm{pVO}_{2} \max \Delta=+2.6 \mathrm{ml} \mathrm{min}^{-1}, 95 \%$ CI $1.2-4.0 p=0.001 ; \mathrm{O}_{2}$ pulse $\Delta=+1.4 \mathrm{ml}_{\text {beat }}{ }^{-1} 95 \%$ CI $\left.0.5-2.3 p=0.001\right)$. Patients with higher baseline fitness completed more physical activity. Regression analyses found adherence was associated with improvement in fitness immediately before surgery $(p=0.048)$, and the amount of physical activity completed was associated with the risk of post-operative pneumonia $(p=0.035)$. Conclusion Pre-operative exercise can maintain cardiorespiratory fitness during NAC and facilitate an increase in fitness before surgery. Greater exercise volumes were associated with a lower risk of post-operative pneumonia, highlighting the importance progressing exercise programmes throughout prehabilitation. Patients with high baseline fitness completed more physical activity and may require less supervision to reach their exercise goals. Further research is needed to explore stratified approaches to prehabilitation.
\end{abstract}

Keywords Oesophageal cancer $\cdot$ Exercise therapy $\cdot$ Pre-operative care $\cdot$ Surgery

\section{Introduction}

Prehabilitation uses the time between diagnosis and surgery to optimise a patient's functional capacity, which is understood

Electronic supplementary material The online version of this article (https://doi.org/10.1007/s11605-020-04561-2) contains supplementary material, which is available to authorized users.

Laura J. Halliday

laura.halliday15@imperial.ac.uk

1 Department of Surgery and Cancer, Imperial College London, London, UK

2 Oesophago-Gastric Cancer Surgery Unit, St. Mary's Hospital, Imperial College Healthcare NHS Trust, London, UK

3 Centre for Active Living, University Centre Shrewsbury/University of Chester, Shrewsbury, Chester, UK to provide a buffer to withstand the physiological stress of surgery. ${ }^{1}$ There is substantial heterogeneity in the content of prehabilitation programmes, but structured exercise is a major component of most studies. $^{2-5}$

Within this rapidly growing area of perioperative medicine, studies have shown that prehabilitation can increase fitness prior to surgery ${ }^{4,6}$ and reduce post-operative complications. $^{7,8}$ Post-operative pneumonia is a wellacknowledged complication after oesophagectomy and is reported in up to $50 \%$ of patients. ${ }^{9-11}$ There are multiple reasons for the particularly high rate of post-operative pulmonary complications following an oesophagectomy: intra-operative one lung ventilation, post-operative diaphragm dysfunction and upper abdominal pain limiting coughing and clearing of secretions. A meta-analysis of pre-operative exercise prior to intra-abdominal surgery found it can reduce the incidence of pulmonary complications by $60 \% .^{7}$ 
Unlike rehabilitation, prehabilitation is strictly time constrained. It is therefore important that exercise behaviour is integrated into the pre-operative period efficiently and effectively. Adherence to exercise is challenging in the preoperative setting. ${ }^{2,4,12}$ A retrospective review of prehabilitation prior to colorectal cancer surgery reported that $40 \%$ of patients did not improve their fitness in response to pre-operative exercise. ${ }^{6}$

Neoadjuvant chemotherapy (NAC) has a negative impact on cardiorespiratory fitness, ${ }^{13,14}$ and adherence to exercise during this time may be particularly challenging. To date, few studies have examined using pre-operative exercise whilst patients are receiving NAC. ${ }^{12,15-17}$

The aims of this study are:

1. To examine pre-operative changes in cardiorespiratory fitness during prehabilitation in oesophageal cancer patients receiving NAC

2. To establish whether adherence to a personalised exercise prescription and the amount of physical activity (PA) completed during prehabilitation are related to cardiorespiratory fitness and the incidence of post-operative pneumonia

3. To characterise how baseline fitness affects the amount of PA completed, changes in fitness and the occurrence of post-operative pneumonia

\section{Materials and Methods}

\section{The PREPARE for Surgery Prehabilitation Programme}

PREPARE (physical activity, respiratory exercises, eat well, psychological well being, ask about medications, remove bad habits, enhanced recovery) for Surgery is a personalised, home-based prehabilitation programme for patients with oesophageal cancer. All patients who were diagnosed with resectable disease were invited to participate in the programme, which started immediately after staging investigations and continued during NAC up to the time of surgery (covering a time period of approximately 16 weeks). Results from all patients who completed the programme and underwent surgery from January 2016 to December 2018 were included in this study.

A home-based exercise programme is agreed with the patient. In keeping with WHO guidelines, patients were prescribed a personalised exercise programme with the aim of trying to achieve a minimum of 600 MET minutes week ${ }^{-1}$ of aerobic exercise, ${ }^{18}$ which equates to $150 \mathrm{~min}$ of moderate/vigorous-intensity activity. Strength exercises were also prescribed alongside aerobic activities. The programme was personalised according to FITT principles (frequency, intensity, time and type $)^{19}$ using the results of submaximal exercise testing and information on activities of daily living, previous exercise behaviour, medical co-morbidities and social circumstances (see Appendix 1 for an example of a personalised exercise programme).

Patients were trained to self-monitor and self-regulate exercise intensity using the Borg scale rating of perceived exertion (RPE) ${ }^{20}$. A weekly telephone touch-point was held with an exercise therapist. Providing the goals were achieved, the exercise programme was progressed in frequency, time and then intensity, according to FITT principles, with the aim achieving a volume of $\geq 1200$ MET minutes week ${ }^{-1}$ or more per week (300 min of moderate/vigorous-intensity activity).

For those who were unable to meet their goals each week, the programme was adapted to their current condition and reevaluated at the next weekly touch-point. Changes were made to the frequency of each exercise, followed by the duration and then type of exercises if further modification was required. Patients also received consultations with clinical nurse specialists if needed. During these sessions, the rationale for prehabilitation was reinforced, potential barriers and facilitators to exercise were explored and motivational interviewing techniques were used to facilitate positive behaviour change. ${ }^{21}$

Following surgery, an enhanced recovery protocol (ERP) was used, with predefined targets for extubation, mobilisation, oral intake, removal of drains and nasogastric tubes and pain control (see Appendix 2). All patients who underwent PREPARE for Surgery followed the same post-operative protocol.

\section{Measurement of Cardiorespiratory Fitness}

Submaximal exercise testing using the Chester Step Test (CST) was used to assess the predicted maximal oxygen uptake $\left(\mathrm{pVO}_{2} \mathrm{max}\right)$ and $\mathrm{O}_{2}$ pulse. ${ }^{22,23}$ Submaximal exercise testing has been used in previous clinical studies and is appropriate for prescription of home-based exercises. ${ }^{24,25}$ The CST was performed by a trained exercise therapist. During the test, patients walked up and down a step at a predefined frequency using a metronome. Three different step heights were used dependent on the patient's abilities. Every $2 \mathrm{~min}$, the speed of the metronome was increased. The test finished when the patient felt unable to continue, was unable to maintain the metronome speed or reached over $70 \%$ of their heart rate reserve.

The CST is a validated method to estimate aerobic power and maximal oxygen uptake $\left(\mathrm{pVO}_{2} \mathrm{max}\right)^{23,26}$ From the $\mathrm{VO}_{2}$ calculated during the CST, $\mathrm{O}_{2}$ pulse was derived. This an indirect representation of stroke volume and demonstrates the central cardiac contribution to overall oxygen uptake. ${ }^{27}$

The CST was undertaken at three time points during prehabilitation: 
- $\quad$ PREPARE $1(\mathrm{P} 1)=$ diagnosis (baseline)

- PREPARE $2(\mathrm{P} 2)=$ completion of NAC. For patients who did not receive NAC this was their baseline measurement (4 to 6 weeks prior to surgery)

- PREPARE 3 (P3) = 1 week prior to surgery

In respect of the known estimate errors of the CST and effects of test-retest familiarity, ${ }^{23,26,28}$ and based on studies examining the clinical effects of improving $\mathrm{pVO}_{2} \mathrm{max}^{29}$ an improvement in fitness was defined as an increase of $10 \%$ or more in $\mathrm{pVO}_{2}$ max.

\section{Measurement of Adherence}

Patients self-reported the frequency, duration and intensity with which they completed each exercise every week using exercise diaries (see Appendix 1). The recorded RPE scores were used to derive the percentage of $\mathrm{VO}_{2}$ max and METSmax at which they exercised, ${ }^{30}$ and thus, using the METSmax derived from the CST, the estimated achieved intensity in METS was calculated. The weekly exercise duration, intensity and frequency for each activity were multiplied to quantify the volume of physical activity (PA) in MET minutes week ${ }^{-1}$. Weekly adherence was calculated as a percentage of actual/ prescribed MET minutes week ${ }^{-1}$.

There is no standardised method for measuring adherence or defining acceptable levels of adherence in exercise studies.

31 We therefore pragmatically defined high adherence as an average weekly adherence of $75 \%$ or greater.

\section{Definition of Pneumonia}

The diagnosis of post-operative pneumonia was defined according to the CDC definition for the clinical diagnosis of a hospital-acquired pneumonia (see Appendix 3). ${ }^{32}$

\section{Statistical Analysis}

Changes in $\mathrm{pVO}_{2}$ max and $\mathrm{O}_{2}$ pulse during prehabilitation were assessed using a repeated-measures one-way analysis of variance (ANOVA) for patients who underwent NAC and had three measurements, and a paired $T$ test for those who did not have NAC and therefore had two measurements. If significant difference was determined with the ANOVA, post hoc paired $T$ tests with a Bonferroni correction were used to determine where the paired differences occurred.

Multivariate analyses of the factors associated with improvement in fitness and with post-operative pneumonia were performed using binary logistic regression. Chi-squared tests were used to establish the effects of an increase in fitness and high adherence on the rate of post-operative pneumonia. Student's $T$ tests were used to compare baseline fitness in patients with high adherence to the rest of the cohort and to compare baseline fitness according to changes in fitness during prehabilitation. To assess the relationship between baseline fitness and average weekly PA, a Pearson correlation test was used. Finally, to assess the variability of self-reported exercise adherence, intra and inter-person coefficient of variances were calculated.

Two-tailed tests were used throughout with a significance level of $p<0.05$. Statistical analysis was performed using SPSS version 25 (IBM, New York, USA).

\section{Results}

Between January 2016 and December 2018, 79 patients with oesophageal or gastro-oesophageal junctional adenocarcinoma entered the PREPARE for Surgery programme. Ten patients did not complete the programme due to a change in clinical status precluding resection (disease progression, development of metastases or deterioration in medical co-morbidities). One patient declined to participate in the programme, and one patient declined surgery after starting treatment. The remaining 67 patients who completed the programme and underwent surgery were included in this study. Characteristics for these patients are shown in Table 1. Of the 67 patients, 60 underwent NAC and had three pre-operative assessments of fitness (P1, P2 and P3). The remaining seven patients did not receive NAC and therefore had two preoperative assessments (P2 and P3).

Eleven patients had a three-stage oesophagectomy and 56 had a two-stage procedure. Both the abdominal and thoracic components were performed as open procedures in 62 patients (93\%); five patients had a laparoscopic abdominal phase followed by an open thoracotomy.

\section{Physical Activity and Adherence}

The overall mean PA was 989 MET minutes week $^{-1}$ (S.D. 805); between P1 and P2, this was 848 MET minutes week ${ }^{-1}$ (S.D 667), and this rose to 1228 MET minutes week $^{-1}$ (S.D 1236) between P2 and P3. Coefficient of variance for selfreported adherence within individual patients was $49 \%$ (intra-person variability) and between patients was $38 \%$ (inter-person variability).

\section{Changes in Fitness during Prehabilitation}

In patients who received $\mathrm{NAC}$, both $\mathrm{pVO}_{2}$ max and $\mathrm{O}_{2}$ pulse changed significantly during prehabilitation (Tables 2 and 3 respectively). $\mathrm{pVO}_{2} \max$ and $\mathrm{O}_{2}$ pulse were preserved between $\mathrm{P} 1$ and $\mathrm{P} 2$, followed by a significant increase between $\mathrm{P} 2$ and P3: $\Delta \mathrm{pVO}_{2} \max =+2.6 \mathrm{ml} \mathrm{min}^{-1} \mathrm{~kg}^{-1}$ (95\% CI 1.24.0); $\Delta \mathrm{O}_{2}$ pulse $=+1.4 \mathrm{ml} \mathrm{beat}^{-1}(95 \%$ CI $0.5-2.3)$. 
Table 1 Study participant characteristics

\begin{tabular}{|c|c|}
\hline \multicolumn{2}{|l|}{ Characteristics } \\
\hline Age - mean (S.D.) & $66(9.7)$ \\
\hline ASA grade $-n(\%)$ ASA 1 & 0 \\
\hline ASA 2 & $57(85 \%)$ \\
\hline ASA 3 & $10(15 \%)$ \\
\hline ASA 4 & 0 \\
\hline Charlson Comorbidity Index score - mean (range) & $5(2-8)$ \\
\hline Pre-operative T stage $-n(\%) \mathrm{T} 1$ & $5(8 \%)$ \\
\hline $\mathrm{T} 2$ & $11(16 \%)$ \\
\hline $\mathrm{T} 3$ & $44(66 \%)$ \\
\hline $\mathrm{T} 4$ & $7(10 \%)$ \\
\hline Pre-operative $\mathrm{N}$ stage $-\mathrm{n}(\%) \mathrm{N} 0$ & $23(34 \%)$ \\
\hline N1 & $33(50 \%)$ \\
\hline $\mathrm{N} 2$ & $8(12 \%)$ \\
\hline N3 & $3(4 \%)$ \\
\hline $\mathrm{NAC}-n(\%)$ & $60(90 \%)$ \\
\hline Baseline weight—mean $\mathrm{kg}$ (S.D.) & $79.7(19.4)$ \\
\hline Baseline $\mathrm{pVO}_{2} \mathrm{max}$-mean (S.D.) & $23.8(6.4)$ \\
\hline Adherence-mean \% (S.D) & \\
\hline Overall & $64 \%(30.1)$ \\
\hline P1-2 & $56 \%(30.1)$ \\
\hline P2-P3 NAC patients & $65 \%(36.3)$ \\
\hline P2-3 non-NAC patients & $85 \%(22.5)$ \\
\hline Post-operative pneumonia $-n(\%)$ & $22(33 \%)$ \\
\hline Overall complications- $n(\%)$ & $44(66 \%)$ \\
\hline Length of stay-median days (IQR) & $10(8-16)$ \\
\hline
\end{tabular}

S.D. standard deviation, $I Q R$ interquartile range

In the whole cohort (including those who did not undergo NAC), both $\mathrm{pVO}_{2}$ max and $\mathrm{O}_{2}$ pulse increased significantly between $\mathrm{P} 2$ and $\mathrm{P} 3: \Delta \mathrm{pVO}_{2} \mathrm{max}=+3.0 \mathrm{ml} \mathrm{min}^{-1} \mathrm{~kg}^{-1}(95 \%$ $\mathrm{CI}=2.0-4.0) ; \Delta \mathrm{O}_{2}$ pulse $=+1.6 \mathrm{ml}^{\text {beat }^{-1}}(95 \% \mathrm{CI}=0.9$ 2.2).

\section{Factors Predictive of Improvement in Cardiorespiratory Fitness Between P1 and P2}

Twenty-one per cent of patients increased their $\mathrm{pVO}_{2}$ max by $10 \%$ or more between P1 and P2. There was no significant relationship between the increase in fitness between P1 and P2 and age, pre-operative stage, baseline fitness, adherence or average weekly PA completed between P1 and P2 $(p>0.05)$.

\section{Factors Predictive of Improvement in Cardiorespiratory Fitness Between P2 and P3}

Fifty-two per cent of patients increased their $\mathrm{pVO}_{2}$ max by $10 \%$ or more between P2 and P3. Adherence with prescribed exercise was significantly associated with the chance of increasing $\mathrm{pVO}_{2}$ max between $\mathrm{P} 2$ and $\mathrm{P} 3$ (Table 4; $p=0.048$ ). There was no significant effect of age, the use of NAC, preoperative stage, baseline fitness or average weekly PA completed between $\mathrm{P} 2$ and $\mathrm{P} 3$.

\section{Factors Predictive of Post-Operative Pneumonia}

Baseline characteristics, adherence, PA, changes in fitness and ERP compliance for patients who developed post-operative pneumonia are shown in Table 5. Average weekly PA over the whole programme was significantly associated with the risk of post-operative pneumonia (Table $6 ; p=0.035$ ). There was no significant effect of age, the use of NAC, pre-operative stage, baseline fitness or adherence to prescribed exercise.

Patients with $\geq 75 \%$ adherence had a lower incidence of post-operative pneumonia, although this difference did not reach statistical significance $(22 \%$ vs $39 \%, p=0.192$; Fig. 1). Patients who increased $\mathrm{pVO}_{2}$ max between $\mathrm{P} 1$ and P2 had a lower incidence of post-operative pneumonia than patients who either maintained or had a fall in $\mathrm{pVO}_{2} \max (9 \%$ vs $41 \%, p=0.045$; Fig. 2). There was no significant difference in the incidence of pneumonia between patients who increased their $\mathrm{pVO}_{2}$ max between $\mathrm{P} 2$ and $\mathrm{P} 3$ and those who did not ( $32 \%$ vs $34 \%, p=0.855)$.

\section{Baseline Fitness and Adherence}

Patients who had $\geq 75 \%$ adherence had a higher baseline $\mathrm{pVO}_{2}$ max than those with less than $75 \%$ adherence (26.1 vs $22.3, p=0.028$; Fig. 3 ). There was also a moderate correlation between baseline $\mathrm{pVO}_{2}$ max and average weekly PA completed throughout the programme, with higher levels of $\mathrm{PA}$ in patients with a higher baseline fitness $(r=0.340, p=0.008)$.

Table $2 \quad \mathrm{pVO}_{2}$ max during prehabilitation

$\mathrm{pVO}_{2}$ max at $\mathrm{P} 1\left(\mathrm{ml} \mathrm{min}{ }^{-1} \mathrm{~kg}^{-1}\right) \quad \mathrm{pVO}_{2} \max$ at $\mathrm{P} 2\left(\mathrm{ml} \mathrm{min}^{-1} \mathrm{~kg}^{-1}\right) \quad \mathrm{pVO} 2{ }_{2}$ max at $\mathrm{P} 3\left(\mathrm{ml} \mathrm{min}^{-1} \mathrm{~kg}^{-1}\right) \quad p$ value

Patients receiving neoadjuvant therapy

$$
24.3 \text { (22.5-26.2; S.D. 6.5) }
$$

Post hoc analysis $24.3(22.5-26.2)$

$$
\begin{aligned}
& 23.2(21.4-25.0 ; \text { S.D. } 6.3) \\
& 23.2(21.4-25.0) \\
& 23.2(21.4-25.0)
\end{aligned}
$$

$23.0(21.3-24.6$; S.D. 6.3$)$

$\begin{array}{ll}25.8(24.0-27.5 ; \text { S.D. } 6.1) & 0.01 \\ 25.8(24.0-27.5) & 0.292 \\ & 0.001\end{array}$

$25.9(24.3-27.5 ;$ S.D. 6.2$)$ 0.001

Results displayed as mean (95\% confidence interval; S.D.)

n/a not applicable, S.D. standard deviation 
Table $3 \quad \mathrm{O}_{2}$ pulse during prehabilitation

$\mathrm{O}_{2}$ pulse at $\mathrm{P} 1\left(\mathrm{ml} \mathrm{beat}^{-1}\right) \quad \mathrm{O}_{2}$ pulse at $\mathrm{P} 2\left(\mathrm{ml} \mathrm{beat}^{-1}\right) \quad \mathrm{O}_{2}$ pulse at $\mathrm{P} 3\left(\mathrm{ml} \mathrm{beat}^{-1}\right) \quad p$ value

Patients receiving neoadjuvant therapy

$$
\begin{aligned}
& 14.7(13.7-15.7 ; \text { S.D. } 3.3) \\
& 14.7(13.7-15.7)
\end{aligned}
$$

Post hoc analysis

Whole cohort

\author{
$14.1(13.0-15.1$; S.D. 3.4) \\ $14.1(13.0-15.1)$ \\ $14.1(13.0-15.1)$
}

$13.9(13.30-14.8 ;$ S.D. 3.3)

$\begin{array}{ll}15.5(14.4-16.5 ; \text { S.D. } 3.5) & 0.002 \\ 15.5(14.4-16.5) & 0.264 \\ & 0.001\end{array}$

$15.4(14.5-16.3$ S.D.3.4)

0.001

Results displayed as mean (95\% confidence interval; S.D.)

n/a not applicable, S.D. standard deviation

\section{Baseline Fitness and Changes in Fitness}

Patients who increased their $\mathrm{pVO}_{2}$ max between $\mathrm{P} 1$ and $\mathrm{P} 2$ had a lower baseline fitness than those who either maintained or had a fall in $\mathrm{pVO}_{2}$ max during this time (19.6 vs 25.4, $p=$ 0.006; Fig. 4). Baseline $\mathrm{pVO}_{2} \max$ was comparable between patients who did and did not increase their fitness between P2 and P3 (23.5 vs 24.4, $p=0.620)$.

\section{Baseline Fitness and Pneumonia}

There was no significant difference in baseline $\mathrm{pVO}_{2}$ max between patients who did and did not develop a post-operative pneumonia (23.9 vs 23.7, $p=0.903)$.

\section{Discussion}

In this 3-year retrospective analysis of patients with oesophageal cancer, we have demonstrated that with prehabilitation, cardiorespiratory fitness can be maintained during NAC and this is then followed by an increase in fitness prior to surgery. Whilst a recovery in fitness following NAC could be anticipated, without prehabilitation the patient is recovering after a substantial fall in fitness from their baseline. ${ }^{13,14}$ We have seen that starting prehabilitation once the decision for curative surgery is made can counteract the effects of chemotherapy and facilitate an increase in exercise intensity just prior to surgery.

Oesophageal cancer surgery has a high post-operative morbidity; the overall incidence of complications often exceeds $70 \%$ and post-operative pneumonia rates of up to $50 \%$ are reported. $^{9-11,33}$ In this study, we have demonstrated that the amount of PA completed was significantly associated with the risk of pneumonia, with the incidence reducing as the volume of PA in MET min week ${ }^{-1}$ increases. Patients should be encouraged to be as active as possible, with review and escalation of programmes to increase PA throughout prehabilitation.

Adherence to the personalised exercise programme was related to improvements in fitness in the period immediately prior to surgery. We found a mean increase in $\mathrm{pVO}_{2}$ max of $2.6 \mathrm{ml} \mathrm{min}^{-1} \mathrm{~kg}^{-1}$ after NAC and $2.9 \mathrm{ml} \mathrm{min}^{-1} \mathrm{~kg}^{-1}$ amongst the whole cohort, which is comparable to changes seen in other prehabilitation studies. ${ }^{34}$ Changes of this magnitude are associated with benefits in other clinical situations. ${ }^{29,35}$

Reported rates of adherence to prehabilitation exercise programmes vary from 16 to $97 \%,{ }^{4}$ with an average of $70 \%$ adherence in home-based programmes. ${ }^{36}$ To date, there is no standardised way to assess adherence to exercise. We have calculated a weekly adherence percentage for each patient,
Table 4 Factors associated with improvement in $\mathrm{pVO}_{2} \max$

\begin{tabular}{|c|c|c|c|c|}
\hline Variable & Odds ratio $(95 \% \mathrm{CI})$ & $\mathrm{B}$ & SE & $p$ value \\
\hline NAC & $0.16(0.00$ to 2.28$)$ & -4.14 & 2.54 & 0.999 \\
\hline Age & 0.98 (0.88 to 1.09$)$ & -0.18 & 0.56 & 0.752 \\
\hline Charlson comorbidity index & $1.63(0.67$ to 4.02$)$ & 0.49 & 0.46 & 0.286 \\
\hline Clinical stage (1) & $0.15(0.00$ to 40.96$)$ & -1.89 & 27.7 & 0.999 \\
\hline Clinical stage (2) & $0.10(0.01$ to 1.37$)$ & -2.34 & 1.36 & 0.840 \\
\hline Clinical stage (3) & 0.49 (0.84 to 2.82$)$ & -0.72 & 0.90 & 0.423 \\
\hline Baseline $\mathrm{pVO} 2 \max \left(\mathrm{ml} \mathrm{min}{ }^{-1} \mathrm{~kg}^{-1}\right)$ & $0.99(0.88$ to 1.10$)$ & -0.15 & 0.59 & 0.795 \\
\hline Total adherence $(\%)$ & 49.38 (1.04 to 2356.0$)$ & 3.90 & 1.97 & 0.048 \\
\hline Average PA (MET min week ${ }^{-1}$ ) & $0.99(0.99$ to 1.00$)$ & -0.002 & 0.001 & 0.126 \\
\hline
\end{tabular}
between P2 and P3 
Table 5 Characteristics of patients who did and did not develop post-operative pneumonia
Table 6 Factors associated with development of post-operative pneumonia

\begin{tabular}{|c|c|c|c|}
\hline Characteristics & $\begin{array}{l}\text { Patients who developed } \\
\text { pneumonia }\end{array}$ & $\begin{array}{l}\text { Patients who did not } \\
\text { develop pneumonia }\end{array}$ & $p$ value \\
\hline$N(\%)$ & $22(33 \%)$ & $45(67 \%)$ & \\
\hline Age - mean (S.D.) & $68.4(10.0)$ & $65.3(9.4)$ & 0.214 \\
\hline Pre-operative T stage $-n(\%)$ & & & 0.725 \\
\hline $\mathrm{T} 1$ & $2(9 \%)$ & $3(7 \%)$ & \\
\hline $\mathrm{T} 2$ & $3(14 \%)$ & $9(20 \%)$ & \\
\hline $\mathrm{T} 3$ & $15(68 \%)$ & $28(62 \%)$ & \\
\hline $\mathrm{T} 4$ & $2(9 \%)$ & $5(11 \%)$ & \\
\hline Pre-operative $\mathrm{N}$ stage $-n(\%)$ & & & 0.494 \\
\hline No & $5(22 \%)$ & $18(40 \%)$ & \\
\hline $\mathrm{N} 1$ & $13(59 \%)$ & $19(43 \%)$ & \\
\hline $\mathrm{N} 2$ & $3(14 \%)$ & $6(13 \%)$ & \\
\hline $\mathrm{N} 3$ & $1(5 \%)$ & $2(4 \%)$ & \\
\hline $\mathrm{NAC}-n(\%)$ & $19(86 \%)$ & $41(91 \%)$ & 0.551 \\
\hline Baseline $\mathrm{pVO}_{2} \mathrm{max}$ - mean (S.D.) & $23.9(5.4)$ & $23.7(6.8)$ & 0.911 \\
\hline \multicolumn{4}{|l|}{ Change in $\mathrm{pVO}_{2} \max -$ mean (S.D.) } \\
\hline$P 1-2$ & $-9.9(16.7)$ & $3.3(27.6)$ & 0.068 \\
\hline P2-P3 & $18.1(22.8)$ & $14.1(17.8)$ & 0.459 \\
\hline \multicolumn{4}{|l|}{ Adherence-mean \% (S.D.) } \\
\hline Overall & $62 \%(26.3)$ & $65 \%(32.0)$ & 0.807 \\
\hline $\mathrm{P} 1-2$ & $55 \%(34.0)$ & $57 \%(22.2)$ & 0.790 \\
\hline P2-P3 & $67 \%(36.7)$ & $69 \%(32.6)$ & 0.813 \\
\hline \multicolumn{4}{|c|}{ Weekly PA-mean MET min week ${ }^{-1}$ (S.D.) } \\
\hline Overall & $785(528)$ & $1084(895)$ & 0.184 \\
\hline $\mathrm{P} 1-2$ & $653(349)$ & $960(779)$ & 0.157 \\
\hline P2-P3 & 954 (699) & $1332(1381)$ & 0.334 \\
\hline Laparoscopic abdominal phase $-n(\%)$ & $2(9 \%)$ & $3(7 \%)$ & 0.723 \\
\hline \multicolumn{4}{|l|}{$\begin{array}{l}\text { Compliance with enhanced recovery } \\
\text { protocol }-n(\%)\end{array}$} \\
\hline Mobilization & $4(18 \%)$ & $19(42 \%)$ & 0.094 \\
\hline Nasogastric tube removal & $7(32 \%)$ & $31(69 \%)$ & 0.006 \\
\hline Drain removal & $5(23 \%)$ & $27(60 \%)$ & 0.006 \\
\hline Oral intake & $1(5 \%)$ & $24(53 \%)$ & 0.001 \\
\hline Pain control & $11(50 \%)$ & $27(60 \%)$ & 0.492 \\
\hline Day 0 extubation & $14(64 \%)$ & $33(73 \%)$ & 0.577 \\
\hline
\end{tabular}

\begin{tabular}{llrrr}
\hline Variable & Odds ratio $(95 \%$ CI $)$ & B & SE & $p$ value \\
\hline NAC & $0.21(0.01$ to 28.32$)$ & -1.57 & 2.51 & 0.531 \\
Age & $1.03(0.92$ to 1.15$)$ & 0.31 & 0.57 & 0.588 \\
Charlson comorbidity index & $1.35(0.62$ to 2.91$)$ & 0.30 & 0.39 & 0.449 \\
Clinical stage (1) & $3.11(0.13$ to 734.1$)$ & 1.14 & 2.79 & 0.684 \\
Clinical stage (2) & $0.20(0.13$ to 3.28$)$ & -1.59 & 1.42 & 0.262 \\
Clinical stage (3) & $0.87(0.14$ to 5.33$)$ & -1.42 & 0.93 & 0.878 \\
Baseline pVO2max & $1.11(0.99$ to 1.25$)$ & 0.10 & 0.61 & 0.083 \\
Total adherence (\%) & $24.23(0.82$ to 715.6$)$ & 3.19 & 1.73 & 0.065 \\
Average PA (MET min week $\left.{ }^{-1}\right)$ & $0.99(0.95$ to 0.99$)$ & -0.02 & 0.01 & 0.035 \\
\hline
\end{tabular}


Fig. 1 Incidence of postoperative pneumonia in patients with high adherence

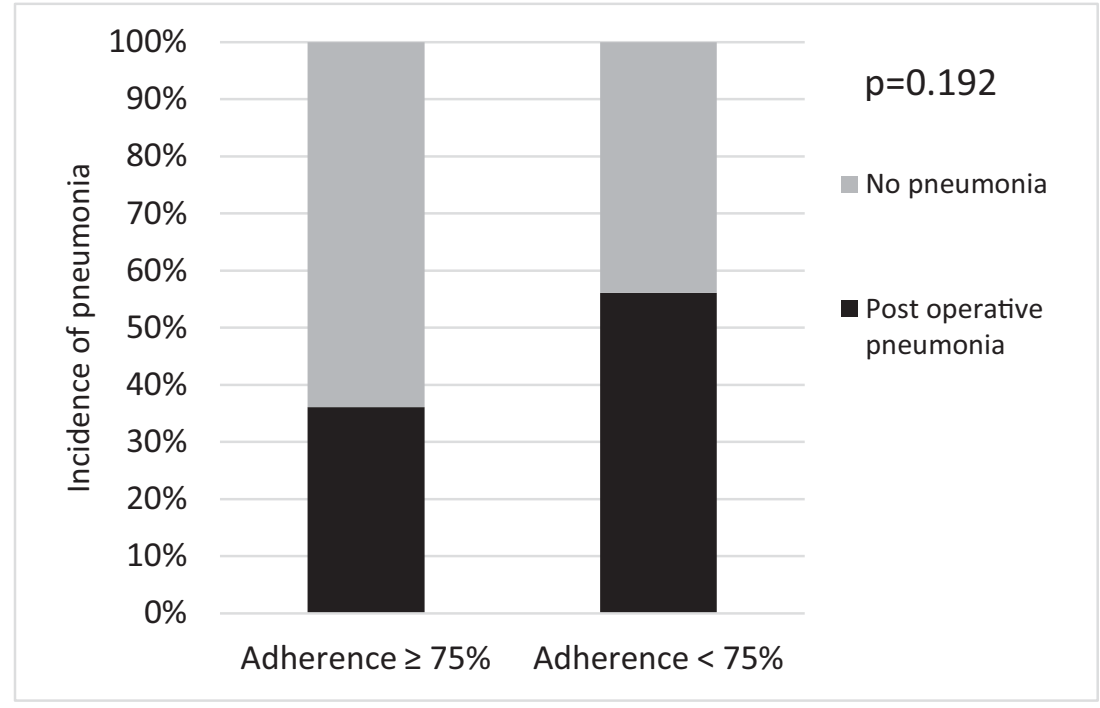

which not only assesses frequency and/or time spent exercising but includes the relative intensity of the exercise and therefore gives a more detailed reflection of the true exercise volume. ${ }^{31}$

NAC had a negative impact on adherence, with an average adherence of $56 \%$. Following NAC, average adherence increased to $65 \%$ but remained lower than the $85 \%$ seen in patients who did not receive NAC. The adherence percentage does not reflect the dose of exercise, as the amount of PA prescribed varied from patient-to-patient. Whilst adherence was challenging for patients during NAC, the average amount of PA achieved exceeded the WHO recommendations of 600 MET minutes week ${ }^{-1}{ }^{18}$ In the 4 - to 6 -week period before surgery, the average weekly PA level increased by over $40 \%$ to more than twice the WHO target. However, further research is still needed to better understand and evaluate ways to support and encourage behaviour change, especially during NAC.

The effect of prehabilitation upon compliance with ERPs is not known. We found low levels of ERP compliance in our patient population and for half of the ERP elements, the compliance was significantly lower in patients who developed post-operative pneumonia. This is unsurprising as poor ERP compliance may be both a consequence of post-operative complications, as well as a risk factor. ERPs should be seen as a continuum of prehabilitation, and efforts to integrate the two pathways may have a positive impact on ERP compliance.

Patients with a low baseline fitness were more likely to increase their fitness during NAC. In parallel to this, those with higher baseline fitness completed more PA and had higher adherence to exercise during prehabilitation. These patients may require less supervision and support to reach their exercise and fitness goals. This raises the possibility of a stratified approach to prehabilitation; as well as a personalised exercise prescription, the level of supervision could be tailored to the patient. In centralised services, such as oesophagogastric cancer, this could have logistical and financial benefits for both the patient and the healthcare provider by
Fig. 2 Incidence of postoperative pneumonia in patients who increased $\mathrm{pVO}_{2}$ max during chemotherapy

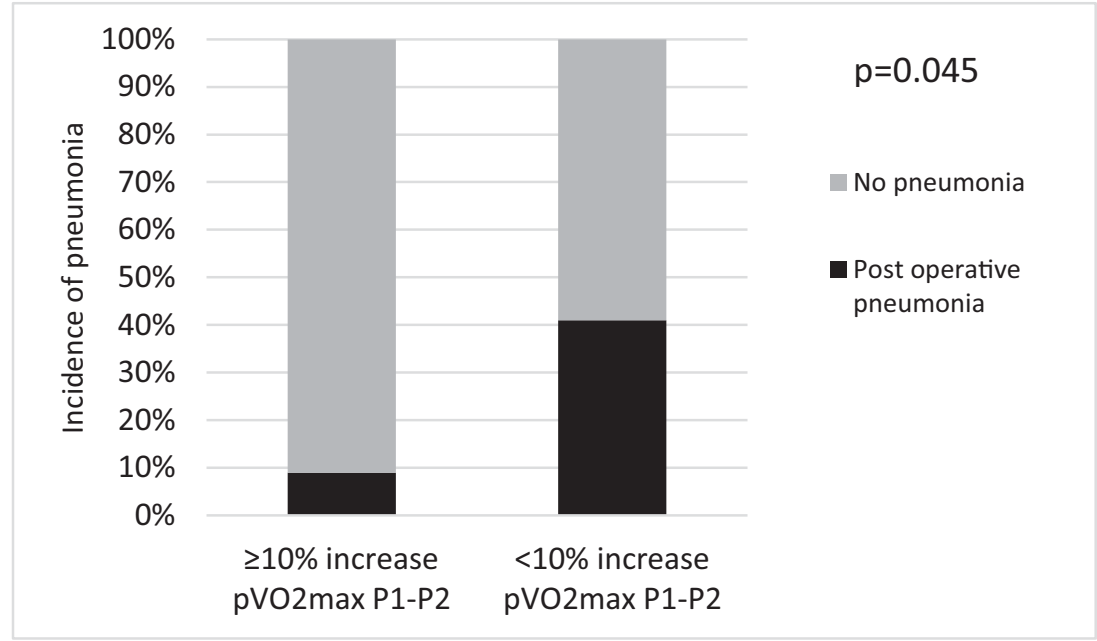


Fig. 3 Baseline fitness in patients with high adherence. Results displayed as mean $( \pm \mathrm{SE})$

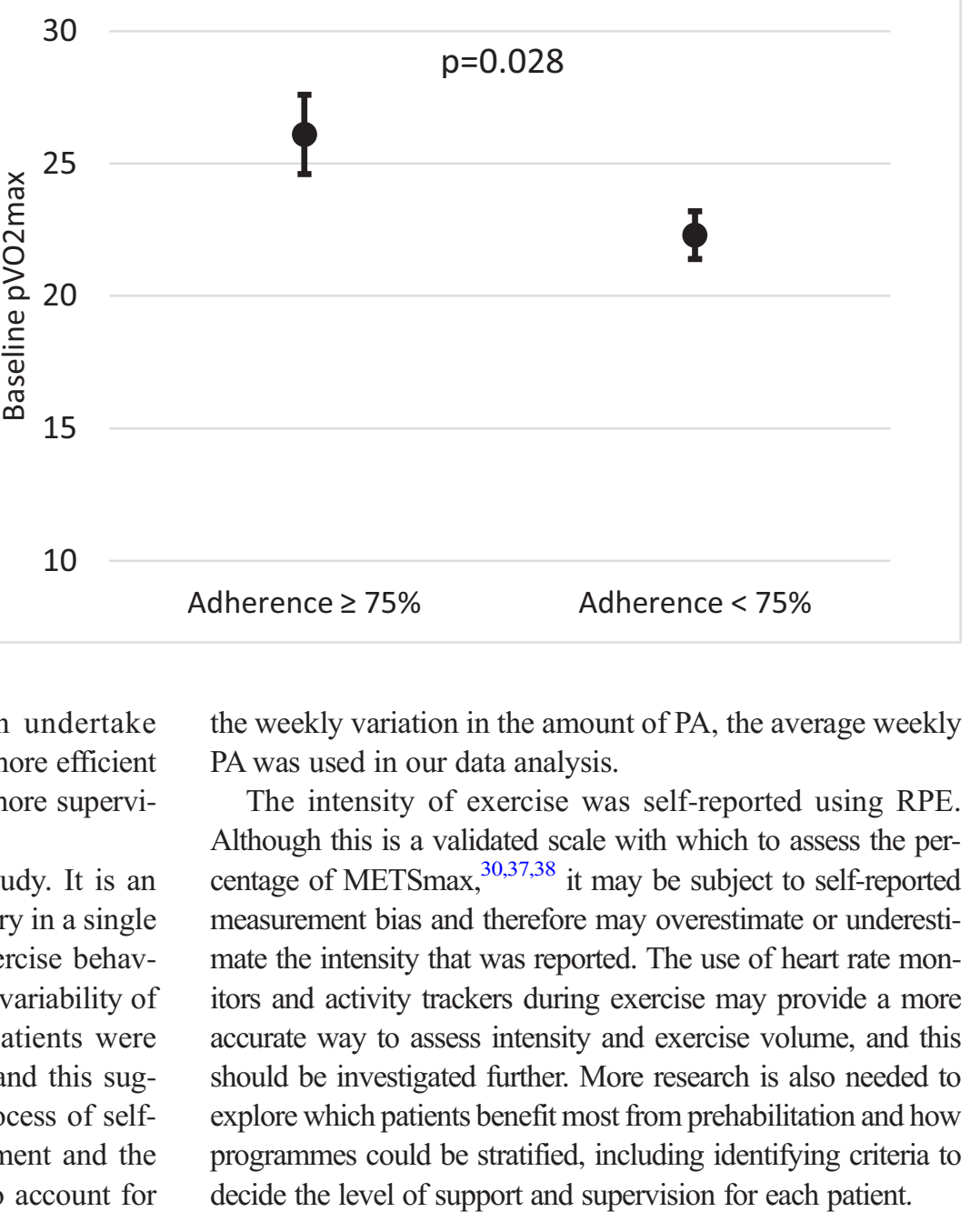

the weekly variation in the amount of PA, the average weekly A was used in our data analysis.

The intensity of exercise was self-reported using RPE. Although this is a validated scale with which to assess the percentage of METSmax, ${ }^{30,37,38}$ it may be subject to self-reported measurement bias and therefore may overestimate or underestimate the intensity that was reported. The use of heart rate montors and activity trackers during exercise may provide a more accurate way to assess intensity and exercise volume, and this should be investigated further. More research is also needed to programmes could be stratified, including identifying criteria to decide the level of support and supervision for each patient. minimising disruption for patients who can undertake prehabilitation independently and generating a more efficient use of resources to support patients who need more supervision to achieve their exercise goals.

There are a number of limitations to this study. It is an observational study of patients undergoing surgery in a single centre. Adherence is based on self-reported exercise behaviour. We saw high inter-patient and intra-patient variability of reported exercise levels, demonstrating that patients were reporting different amounts of PA each week, and this suggests that they were actively engaged in the process of selfreporting. However, it is a subjective measurement and the accuracy of these reports cannot be verified. To account for
Fig. 4 Baseline fitness in patients who increased their fitness. Results displayed as mean $( \pm \mathrm{SE})$

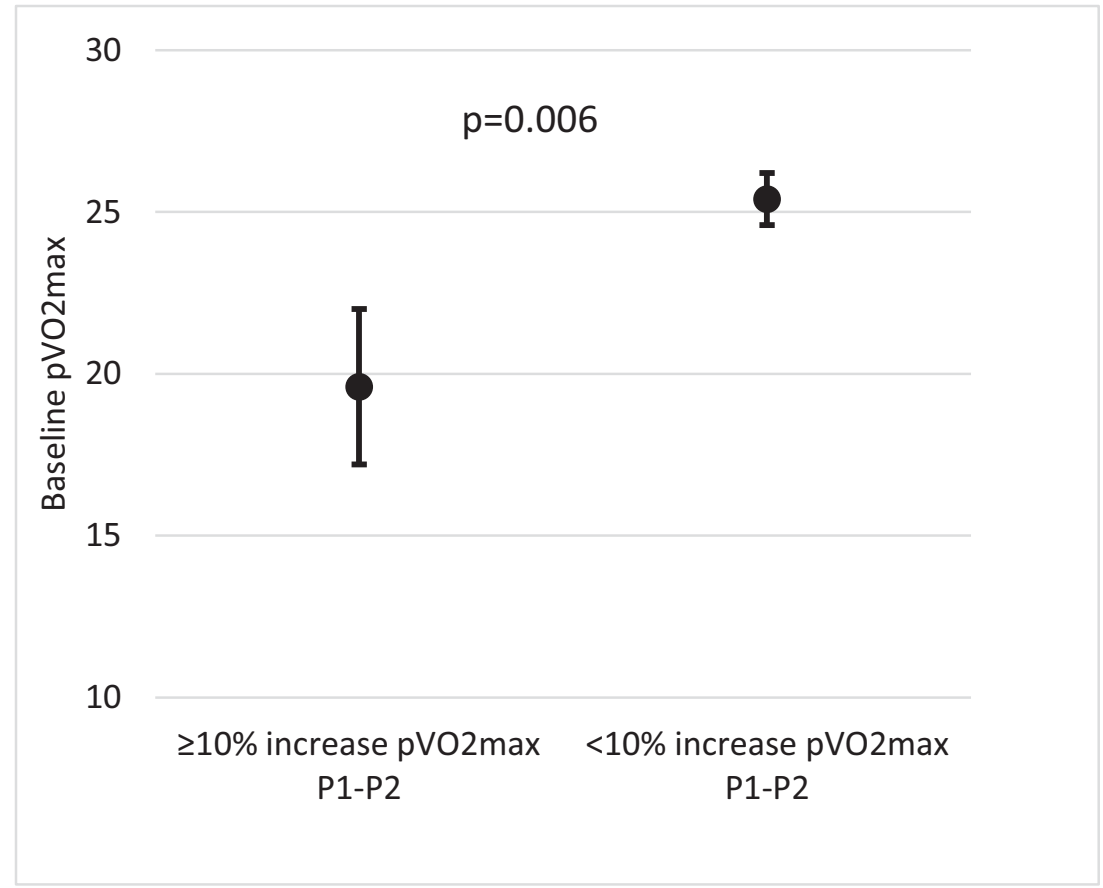




\section{Conclusion}

The time available to improve a patient's fitness prior to surgery is tightly limited, and therefore, it is important to use this time efficiently. We have shown that sustained levels of PA can be achieved during NAC, and this can protect against the impact of chemotherapy on cardiorespiratory fitness. Increasing amounts of PA completed during prehabilitation was associated with a lower risk of post-operative pneumonia. Therefore, we propose that exercise goals should not be seen as a fixed concept during prehabilitation; instead, a personalised approach should encourage patients to continually escalate and maximise their PA to achieve the maximum benefit in the pre-operative period. Patients with higher baseline fitness completed more PA and had higher adherence. Further research is needed to establish which patients benefit most and thus identify potential screening criteria for a stratified approach to the delivery of prehabilitation programmes.

Authors' Contributions All the authors meet the journal's criteria for authorship. Conception and design: LH, JB, VWB, KM. Collection and assembly of data: $\mathrm{LH}, \mathrm{ED}, \mathrm{VWB}, \mathrm{HO}$. Data analysis and interpretation: LH, ED, HO, JB, KM. Manuscript writing: all listed authors. Final approval of manuscript: all listed authors.

\section{Compliance with Ethical Standards}

Conflict of Interest The authors declare that they have no conflict of interest.

Open Access This article is licensed under a Creative Commons Attribution 4.0 International License, which permits use, sharing, adaptation, distribution and reproduction in any medium or format, as long as you give appropriate credit to the original author(s) and the source, provide a link to the Creative Commons licence, and indicate if changes were made. The images or other third party material in this article are included in the article's Creative Commons licence, unless indicated otherwise in a credit line to the material. If material is not included in the article's Creative Commons licence and your intended use is not permitted by statutory regulation or exceeds the permitted use, you will need to obtain permission directly from the copyright holder. To view a copy of this licence, visit http://creativecommons.org/licenses/by/4.0/.

\section{References}

1. Wynter-Blyth V, Moorthy K. Prehabilitation: preparing patients for surgery. BMJ 2017;358. Available from: doi: https://doi.org/10. 1136/bmj.j3702.

2. Minnella EM, Carli F. Prehabilitation and functional recovery for colorectal cancer patients. Eur J Surg Oncol 2018;44:919-26.

3. Zylstra J, Boshier P, Whyte GP, Low DE, Davies AR. Perioperative patient optimization for oesophageal cancer surgery; From prehabilitation to enhanced recovery. Best Pract Res Clin Gastroenterol 2018;36:61-73.

4. Vermillion SA, James A, Dorrell RD, Brubaker P, Mihalko SL, Hill AR, Clark CJ. Preoperative exercise therapy for gastrointestinal cancer patients: a systematic review. Syst Rev 2018;7:1-10.
5. Levett DZH, Edwards M, Grocott M, Mythen M. Preparing the patient for surgery to improve outcomes. Best Pract Res Clin Anaesthesiol 2016;30:145-57.

6. Minnella EM, Bousquet-Dion G, Awasthi R, Scheede-Bergdahl C, Carli F. Multimodal prehabilitation improves functional capacity before and after colorectal surgery for cancer: a five-year research experience. Acta Oncol 2017;56:295-300.

7. Hughes M, Hackney R, Lamb P, Wigmore S, Deans C, Skipworth R. Prehabilitation before Major Abdominal Surgery: A Systematic Review and Meta-Analysis. Int J Surg 2018;55. Available from: doi: https://doi.org/10.1016/j.ijsu.2018.05.692.

8. Moran J, Guinan E, Mccormick P, Larkin J, Mockler D, Hussey J, Moriarty J, Wilson F. The ability of prehabilitation to influence postoperative outcome after intra- abdominal operation: A systematic review and meta-analysis. Surgery 2016;160:1189-201.

9. Paul S, Altorki N. Outcomes in the management of esophageal cancer. J Surg Oncol 2014;110:599-610.

10. Sinclair RCF, Phillips AW, Navidi M, Griffin SM, Snowden CP. Pre-operative variables including fitness associated with complications after oesophagectomy. Anaesthesia 2017;72:1501-7.

11. Goense L, van Rossum PSN, Tromp M, Joore HC, van Dijk D, Kroese AC, Ruurda JP, van Hillegersberg R. Intraoperative and postoperative risk factors for anastomotic leakage and pneumonia after esophagectomy for cancer. Dis Esophagus 2017; 30. Available from: doi: https://doi.org/10.1111/dote.12517.

12. Minnella EM, Awasthi R, Loiselle S, Agnihotram RV, Ferri LE, Carli F. Effect of Exercise and Nutrition Prehabilitation on Functional Capacity in Esophagogastric Cancer Surgery: A Randomized Clinical Trial. JAMA Surg 2018;153:1081-9.

13. Hoppe S, Rainfray M, Fonck M, Hoppenreys L, Blanc J, Ceccaldi J, Mertens C, Blanc-Bisson C, Imbert Y, Cany L, Vogt L, Dauba J, Houede N, Bellera CA, Floquet A, Fabry MN, Ravaud A, Chakiba C, Mathoulin-Pelissier S, Soubeyran P. Functional decline in older patients with cancer receiving first- line chemotherapy. J Clin Oncol 2013;31:3877-82.

14. West MA, Loughney L, Lythgoe D, Barben CP, Sripadam R, Kemp GJ, Grocott MP, Jack S. Effect of prehabilitation on objectively measured physical fitness after neoadjuvant treatment in preoperative rectal cancer patients: a blinded interventional pilot study. $\mathrm{Br} \mathrm{J}$ Anaesth 2015;114:244-51.

15. Ngo-Huang A, Parker NH, Wang X, Petzel M,QB Fogelman D, Schadler KL, Bruera E, Fleming JB, Lee JE, Katz MHG. Homebased exercise during preoperative therapy for pancreatic cancer. Langenbecks Arch Surg 2017;402:1175-85.

16. Dewberry LC, Wingrove LJ, Marsh MD, Glode AE, Schefter TE, Leong S, Purcell WT, McCarter MD. Pilot Prehabilitation Program for Patients With Esophageal Cancer During Neoadjuvant Therapy and Surgery. J Surg Res 2019;235:66-72.

17. Moug SJ, Mutrie N, Barry SJE, Mackay G, Steele RJC, Boachie C, Buchan C, Anderson AS. Prehabilitation is feasible in patients with rectal cancer undergoing neoadjuvant chemoradiotherapy and may minimize physical deterioration: results from the REx trial. Colorectal Dis 2019;21:548-62.

18. World Health Organisation. Global recommendations on physical activity for health. Geneva: World Health Organisation. 2010.

19. American College of Sports Medicine. ACSMs Guidelines for Exercise Testing and Prescription. Philadelphia: Lippincott, Williams \& Wilkins; 2010.

20. Borg G. Borg's Perceived Exertion and Pain Scales. Illinois: Human Kinetics; 1998.

21. Rollnick S, Butler CC, Kinnersley P, Gregory J, Mash B. Motivational interviewing. BMJ 2010;340. Available from: doi: https://doi.org/10.1136/bmj.jc1900.

22. Buckley J, Holmes J, Mapp G. Exercise on Prescription: Activity for Cardiovascular Health, Oxford: Butterworth-Heinemann; 1998. 
23. Sykes K, Roberts A. The Chester step test - a simple yet effective tool for the prediction of aerobic capacity. Physiotherapy 2004;90: 183-8.

24. Du H, Newton PJ, Salamonson Y, Carrieri-Kohlman V, Davidson PM. A Review of the Six- Minute Walk Test: Its Implication as a Self- Administered Assessment Tool. Eur J Cardiovasc Nurs 2009;8:2-8.

25. Grove $\mathrm{T}$. Incremental shuttle walk test in cardiac rehabilitation. $\mathrm{Br} \mathrm{J}$ Cardiac Nurs 2013;8:31-7.

26. Buckley JP, Sim J, Eston RG, Hession R, Fox R. Reliability and validity of measures taken during the Chester step test to predict aerobic power and to prescribe aerobic exercise. Br J Sports Med 2004:38:197-205.

27. Ridgway ZA, Howell SJ. Cardiopulmonary exercise testing: a review of methods and applications in surgical patients. Eur J Anaesthesiol 2010;27:858-65.

28. Nevill AM, Atkinson G. Assessing agreement between measurements recorded on a ratio scale in sports medicine and sports science. Br J Sports Med 1997;31:314-8.

29. Swank MA, Horton J, Fleg JL, Fonarow GC., Keteyian S, Goldberg L, Wolfel G, Handberg EM, Bensimhon D, Illiou MC, Vest M, Ewald G, Blackbrun G. Modest Increase in Peak VO2 Is Related to Better Clinical Outcomes in Chronic Heart Failure Patients: Results From Heart Failure and a Controlled Trial to Investigate Outcomes of Exercise Training. Circ Heart Fail 2012;5:579-85.

30. Buckley J, Jones J. Tables for assessing, monitoring and guiding physical activity/exercise intensity in programmes for cardiovascular disease prevention and rehabilitation. London: British Association for Cardiovascular Prevention and Rehabilitation; 2012.

31. Hawley-Hague H, Horne M, Skelton DA, Todd C. Review of how we should define (and measure) adherence in studies examining older adults' participation in exercise classes. BMJ Open 2016;6.
Available from: doi: https://doi.org/10.1136/bmjopen-2016011560 .

32. Horan T, Gaynes R. Surveillance of noscomial infection. In: Mayhill C, editor. Hospital Epidemiology and Infection Control. Philadelphia: Lippincott Williams \& Wilkins; 2004. p. 1659-702.

33. Low ED, Kuppusamy MK, Alderson D, Cecconello I, Chang CA., Darling G, Davies A, D'Journo XB, Gisbertz SS, Griffin SM, Hardwick R, Hoelscher A, Hofstetter W, Jobe B, Kitagawa Y, Law S, Mariette C, Maynard N, Morse CR, Nafteux P, Pera M, Pramesh CS, Puig S, Reynolds JV, Schroeeder W, Smithers M, Wijnhoven BPL Benchmarking Complications Associated with Esophagectomy. Ann Surg 2019;269:291-8.

34. Dunne DFJ, Jack S, Jones RP, Jones L, Lythgoe DT, Malik HZ, Poston GJ, Palmer DH, Fenwick SW. Randomized clinical trial of prehabilitation before planned liver resection. Br J Surg 2016;103: 504-12.

35. Petrella RJ, Lattanzio CN, Shapiro S, Overend T. Improving aerobic fitness in older adults: effects of a physician-based exercise counseling and prescription program. Can Fam Physician 2010;56:e191-200.

36. Thomas G, Tahir MR, Bongers BC, Kallen VL, Slooter GD, Van Meeteren NL. Prehabilitation before major intra-abdominal cancer surgery: A systematic review of randomised controlled trials. Eur J Anaesthesiol 2019;36:933-45.

37. Borg GA. Psychophysical bases of perceived exertion. Med Sci Sports Exerc 1982;14:377-81.

38. Colberg SR, Swain DP, Vinik AI. Use of heart rate reserve and rating of perceived exertion to prescribe exercise intensity in diabetic autonomic neuropathy. Diabetes Care 2003;26:986-90.

Publisher's Note Springer Nature remains neutral with regard to jurisdictional claims in published maps and institutional affiliations. 